Supporting information for:

\title{
A DFT Study of the Systematic Variations in Metal-Ligand Bond Lengths of Coordination Complexes: The Crucial Role of the Condensed Phase
}

Rosalie K. Hocking ${ }^{1 *}$, Robert J. Deeth ${ }^{2 *}$ and Trevor W. Hambley ${ }^{1}{ }^{*}$ 
<smiles>NCCN[C@@]12NCCN1C21NCCN1</smiles><smiles>CC(C)(C)NC1(NC(C)(C)C)NC(C)(C)NC1(C)C(C)(C)C</smiles>

1) $\left[\mathrm{Co}(\mathrm{en})_{3}\right]^{3+}$ Co ${ }^{\text {III }}-\mathbf{N} 1.961 \AA$

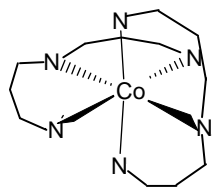

4) $\left[\mathrm{Co}(\mathrm{dpt})_{2}\right]^{3+}$

Co ${ }^{\text {III }}-N$ 1.977 $1.9 \AA$

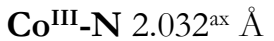

2) $\left[\mathrm{Co}(\text { tmen })_{3}\right]^{3+}$

Co ${ }^{\text {III }}-\mathbf{N} 1.994 \AA$

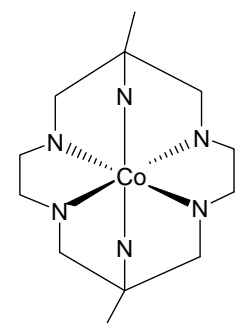

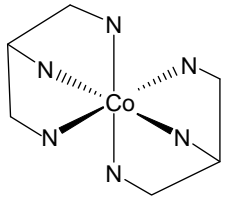

3) $\left[\mathrm{Co}(\operatorname{trap})_{2}\right]^{3+}$

Co ${ }^{\text {III }}-\mathbf{N} 1.943^{\text {eq }} \AA$ Co ${ }^{\text {III }}-\mathbf{N} 1.963^{\text {ax }} \AA$

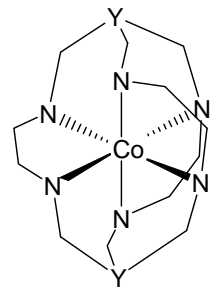

6) $[\mathrm{Co}(\mathrm{sar})]^{3+}$
Figure S1. The series of $\mathrm{Co}^{\mathrm{III}}$ hexaamines referred to in this work. Crystallographic values for the $\mathrm{Co}^{\mathrm{III}}-\mathrm{N}$ bond lengths are given underneath each illustration the references are in the table below.

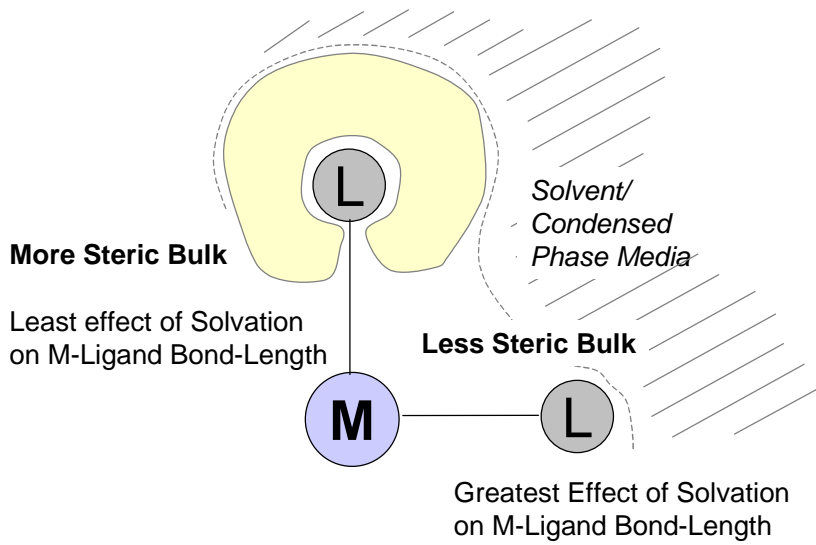

Figure S3. An illustration of how the continuum models effects anionic and neutral donors differently.
Figure S2. A schematic illustration of how steric bulk can shield a metal-ligand interaction from the effects of the environment.

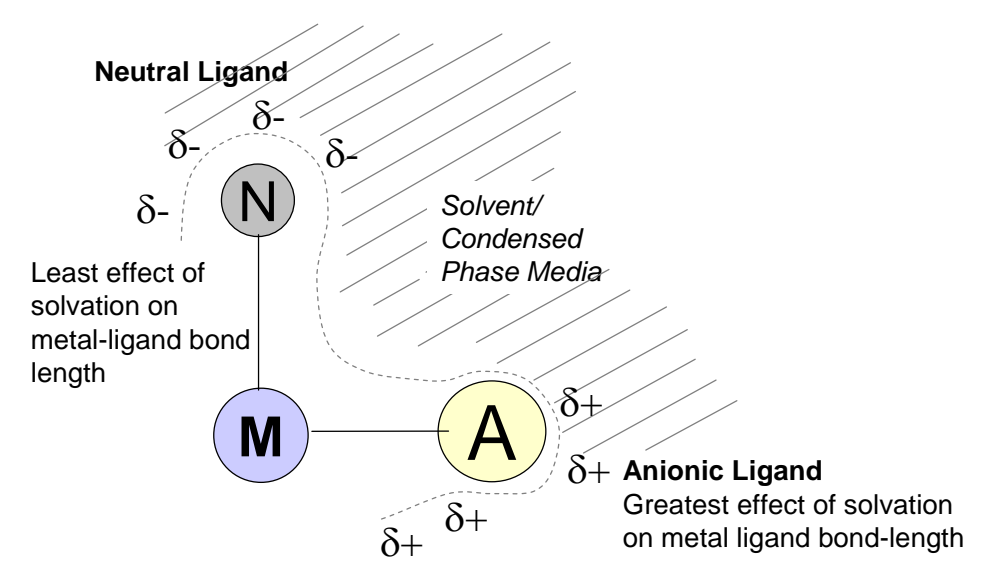


Table S1. Experimental and calculated geometric data for compounds given in Figure 1.

\begin{tabular}{|c|c|c|c|c|c|c|}
\hline Compound & $\begin{array}{l}\text { Experi } \\
\text { mental }\end{array}$ & $\begin{array}{l}\text { Molecular } \\
\text { Mechanics } \\
1\end{array}$ & $\begin{array}{l}L D A \\
V W N\end{array}$ & $\begin{array}{l}\text { LDA } \\
\text { VWN+ } \\
\text { Solvation }\end{array}$ & $\begin{array}{l}\text { Gradient } \\
\text { Corrected } \\
\text { Beck-Perdew }\end{array}$ & $\begin{array}{l}\text { Gradient- } \\
\text { Corrected } \\
\text { Becke-Perdew } \\
\text { +Solvation } \\
\end{array}$ \\
\hline$\left[\mathrm{Co}\left(\mathrm{NH}_{3}\right)_{6}\right]^{3+}$ & 1.963 & 1.964 & 1.963 & 1.925 & 2.030 & 2.004 \\
\hline $\begin{array}{l}{\left[\mathrm{Co}(\mathrm{en})_{3}\right]^{3+}} \\
{\left[\mathrm{Co}(\mathrm{tmen})_{3}\right]^{3+}{ }^{3+}}\end{array}$ & $\begin{array}{l}1.961 \\
1.994\end{array}$ & $\begin{array}{l}1.965 \\
1.989\end{array}$ & $\begin{array}{l}1.958 \\
1.957\end{array}$ & $\begin{array}{l}1.929 \\
1.945\end{array}$ & $\begin{array}{l}2.021 \\
2.016\end{array}$ & $\begin{array}{l}1.987 \\
2.004\end{array}$ \\
\hline$\left[\mathrm{Co}\left(\mathrm{NH}_{2} \mathrm{CH}_{3}\right)_{6}\right]^{3+3}$ & 2.013 & 2.008 & 1.970 & 1.945 & 2.047 & 2.012 \\
\hline$\left[\mathrm{Co}(\operatorname{trap})_{2}\right]^{3+4}$ & $\begin{array}{l}1.943^{\mathrm{eq}} \\
1.963^{\mathrm{ax}}\end{array}$ & $\begin{array}{l}1.937^{\mathrm{eq}} \\
1.960^{\mathrm{ax} .}\end{array}$ & $\begin{array}{l}1.944 \\
1.965\end{array}$ & $\begin{array}{l}1.915^{\mathrm{eq}} \\
1.933^{\mathrm{ax}}\end{array}$ & $\begin{array}{l}1.997 \\
2.018\end{array}$ & $\begin{array}{l}1.961^{\mathrm{eq}} \\
1.983^{\mathrm{ax}}\end{array}$ \\
\hline $\begin{array}{l}{[\mathbf{C o}(\text { trans- }} \\
\text { diammac) }]^{3+}{ }_{4-6} \\
{\left[\mathbf{C o}(\mathbf{d p t})_{2}\right]^{3+}{ }^{7,8}}\end{array}$ & $\begin{array}{l}1.940^{\mathrm{eq}} \\
1.947^{\mathrm{ax}} \\
1.977 \\
2.032\end{array}$ & $\begin{array}{l}1.935^{\mathrm{eq}} \\
1.947^{\mathrm{ax} .} \\
1.976 \\
2.030\end{array}$ & $\begin{array}{l}1.931 \\
1.931 \\
1.970 \\
\\
2.041\end{array}$ & $\begin{array}{l}1.913 \\
1.915 \\
1.943 \\
1.981\end{array}$ & $\begin{array}{l}1.980 \\
1.986 \\
2.037\end{array}$ & $\begin{array}{l}1.961 \\
1.957 \\
1.996\end{array}$ \\
\hline
\end{tabular}




\section{A comparison with organic compounds}

In order to gain a better understanding of the structural changes across a series of metal complexes a comparison was made with an analogous series of organic compounds where a largely structure change is observed. To this end, the series of alkane compounds described in Table 1 was chosen. This series of compounds was originally

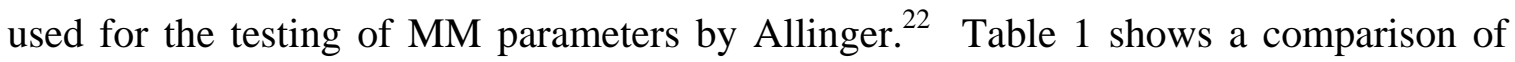
experimental and calculated C-C bond lengths. In all cases the calculated C-C bond lengths are within error of the experimentally calculated C-C bond lengths, indicating that the overestimation of bond lengths is not uniform across all series of compounds. It has been noted elsewhere that the metal-ligand bond lengths of organometallic

compounds are reproduced correctly with the same methods. ${ }^{23}$ This implies there is a fundamental difference in the treatment of Werner-type compounds and organometallic systems by the DFT methods used. We also note that inclusion of solvation nor changes to the dielectric of the environment have much effect on the bond lengths of this series. 
Table 1. Bond lengths in a series of organic compounds with varying steric bulk. ${ }^{22}$

\begin{tabular}{|c|c|c|c|}
\hline \multirow{2}{*}{ Compound } & \multicolumn{3}{|c|}{ C-C Bond Length ( $\AA$ ) } \\
\hline & Experimental & $\begin{array}{c}\text { Calculated } \\
\text { (BP86) }\end{array}$ & $\begin{array}{l}\text { Calculated (BP86) with } \\
\text { Solvation Corrections. } \\
\text { with (Dielectric (78.8)) }\end{array}$ \\
\hline $\mathrm{H}_{3} \mathrm{C}-\mathrm{CH}_{3}$ & 1.532 & 1.532 & $\begin{array}{l}\text { 1.537(78.8); 1.537(10); } \\
\text { 1.534(3); 1.532(1); } \\
\text { 1.532(0.1); }\end{array}$ \\
\hline $\mathrm{CH}_{3} \mathrm{CH}_{2}-\mathrm{CH}_{3}$ & 1.534 & 1.533 & $\begin{array}{l}\text { 1.534(78.8); 1.533(10); } \\
1.533(1)\end{array}$ \\
\hline$\left(\mathrm{CH}_{3}\right)_{2} \mathrm{CH}-\mathrm{CH}_{3}$ & 1.535 & 1.535 & $\begin{array}{l}\text { 1.541(78.8); 1.529(10); } \\
1.536(1)\end{array}$ \\
\hline$\left(\mathrm{CH}_{3}\right)_{3} \mathrm{C}-\mathrm{CH}_{3}$ & 1.539 & 1.540 & $\begin{array}{l}\text { 1.534(78.8); 1.534(10); } \\
1.540(1)\end{array}$ \\
\hline$\left(\mathrm{CH}_{3}\right)_{3} \mathrm{C}-\mathrm{C}\left(\mathrm{CH}_{3}\right)_{3}$ & $1.582^{* *}$ & 1.594 & $\begin{array}{l}\text { 1.583(78.8); 1.585(10): } \\
1.583(3) ; 1.593(1)\end{array}$ \\
\hline$\left(\left(\mathrm{CH}_{3}\right)_{3} \mathrm{C}\right)_{2}-\mathrm{CH}\left(\mathrm{C}\left(\mathrm{CH}_{3}\right)_{3}\right)$ & $1.611^{* *}$ & 1.631 & $\begin{array}{l}\text { Too computationally } \\
\text { expensive. }\end{array}$ \\
\hline
\end{tabular}




\section{Applications of solvation models to other systems}

\section{Comparison of the two series $\left[\mathrm{NiN}_{n}(\mathrm{O}-\mathrm{R})_{6-n}\right]^{2-n}$ and $\left[\mathrm{NiN}_{n}\left(\mathrm{H}_{2} \mathrm{O}\right)_{6-n}\right]^{2+}$ (where, $(\mathrm{O}-$}

\section{R) = anionic oxygen donor)}

It was felt that a number of effects observed in the $\left[\mathrm{PtCl}_{n}\left(\mathrm{NH}_{3}\right)_{4-n}\right]^{2-n}$ and $\left[\mathrm{PtCl}_{n}(\mathrm{CO})_{4-n}\right]^{2-n}, \quad n=0-4$ series were a structural manifestation of the effects of complexes that contain a mixture of anionic and neutral donors. To study this, two subsets of the general series $\left[\mathrm{Ni}^{\mathrm{II}} \mathrm{N}_{n} \mathrm{O}_{6-n}\right]$ studied elsewhere ${ }^{1}$ were separated into the subsets $\left[\mathrm{NiN}_{n}(\mathrm{O}-\mathrm{R})_{6-n}\right]^{4-n}(\mathrm{O}-\mathrm{R}=$ anionic oxygen donor either carboxylate or an alcohol) and $\left[\mathrm{NiN}_{n}\left(\mathrm{H}_{2} \mathrm{O}\right)_{6-n}\right]^{2+}$. These series were chosen for this comparison because whilst one series has a mixture of anionic (O-R) and neutral donors $\left(\mathrm{Nsp}^{3}\right)$, the other has two neutral donors, $\left(\mathrm{H}_{2} \mathrm{O}\right.$ and $\left.\mathrm{Nsp}^{3}\right)$, thus they provided a good test of the above hypothesis. Analysis of the structures contained in these subsets confirms that both obey the general trend of the $\left[\mathrm{Ni}^{\mathrm{II}} \mathrm{N}_{n} \mathrm{O}_{6-n}\right]$ series, described elsewhere. ${ }^{1}$. That is, as the number of nitrogen donors in the coordination sphere increase the $\mathrm{Ni}^{\mathrm{II}}$-O bond lengths increase. ${ }^{1}$

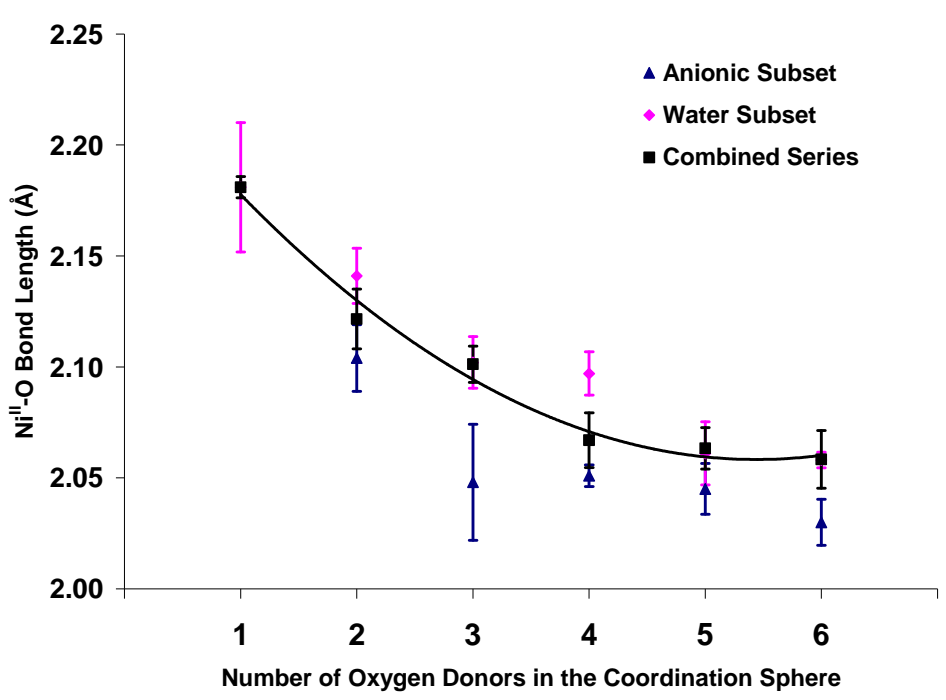

Figure S4. Comparison of statistical analyses of the $\mathrm{Ni}^{\mathrm{II}}-\mathrm{O}$ bond lengths in $\left[\mathrm{Ni}^{\mathrm{II}} \mathrm{N}_{n}(\mathrm{O}-\mathrm{R})_{6-n}\right] \quad$ and $\left[\mathrm{NiN}_{n}\left(\mathrm{H}_{2} \mathrm{O}\right)_{6-n}\right]^{2+}$, with the series described in elwhere 
Figures S3 and S4 compare the calculated and experimental trends in $\mathrm{Ni}^{\mathrm{iI}}$-O bond lengths for the two series, $\left[\mathrm{NiN}_{n}(\mathrm{O}-\mathrm{R})_{6-n}\right]^{n-4}\left[\mathrm{Ni}^{\mathrm{II}} \mathrm{N}_{n}\left(\mathrm{H}_{2} \mathrm{O}\right)_{6-n}\right]^{2+}$. For the series $\left[\mathrm{NiN}_{n}(\mathrm{O}-\mathrm{R})_{6-n}\right]^{n-4}$, the gas phase DFT calculations calculate the reverse trend to those observed experimentally. This is in contrast with the series $\left[\mathrm{Ni}^{\mathrm{II}} \mathrm{N}_{n}\left(\mathrm{H}_{2} \mathrm{O}\right)_{6-n}\right]^{2+}$, where the trends consistent with those observed experimentally are calculated. The trends in both series are calculated correctly following the addition of solvation corrections.

The inconsistencies between the calculations of the series, $\left[\operatorname{NiN}_{n}(\mathrm{O}-\mathrm{R})_{6-n}\right]^{n-4}$ $\left[\mathrm{Ni}^{\mathrm{II}} \mathrm{N}_{n}\left(\mathrm{H}_{2} \mathrm{O}\right)_{6-n}\right]^{2+}$, are analogous to those of the Pt series described in the paper, and provide further evidence that the incorrect structure trends are a consequence of the inability of gas phase-DFT to deal with Werner-Type compounds which contain a mixture of anionic and neutral donors. ${ }^{1}$ 


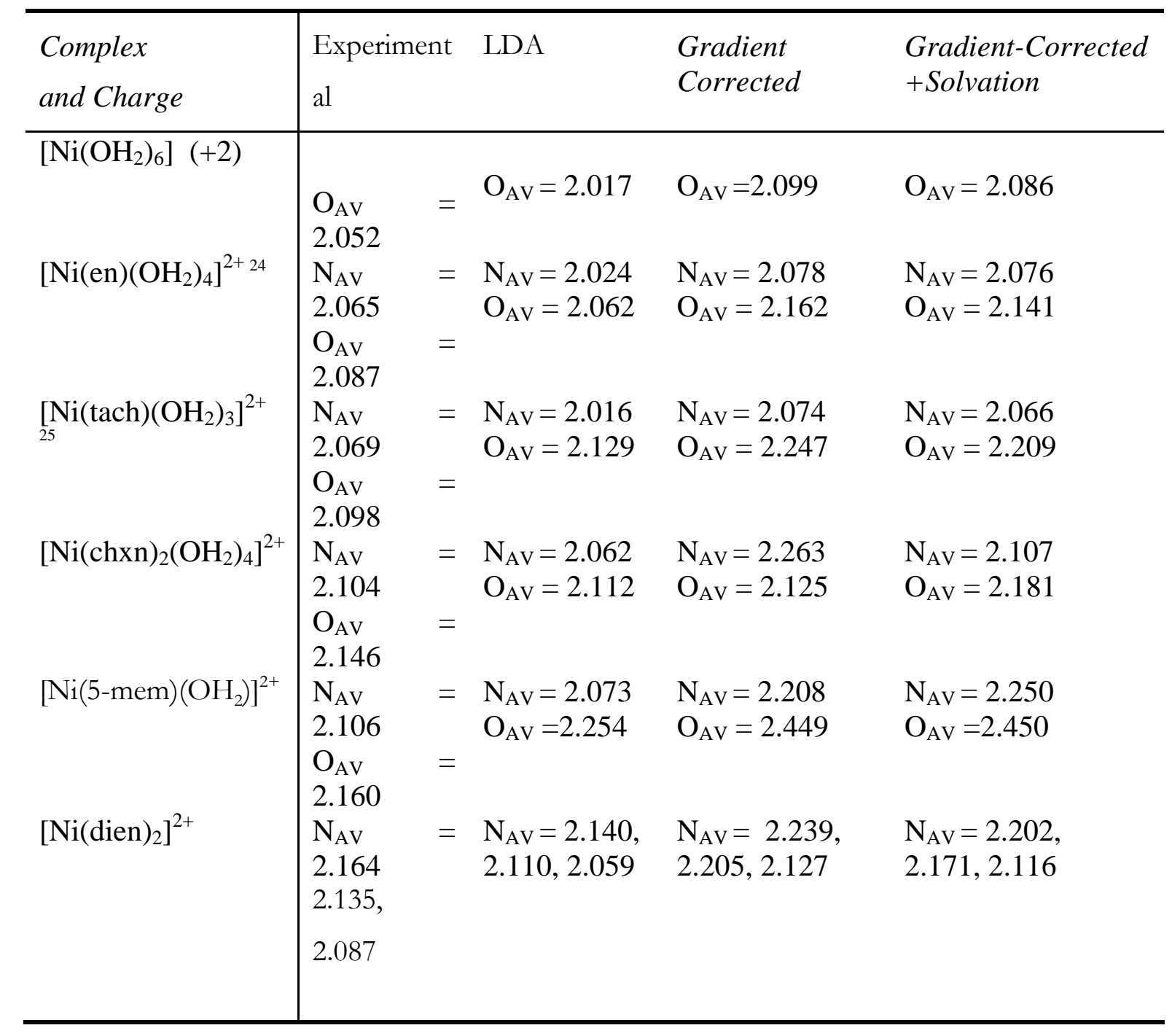


Table S2. Relative performance of ADF calculations on Anionic Nickel series

\begin{tabular}{|c|c|c|c|c|c|c|}
\hline $\begin{array}{l}\text { Complex } \\
\text { and Charge }\end{array}$ & Experimental & LDA & $\begin{array}{l}\text { LDA+ } \\
\text { Solvation }\end{array}$ & $\begin{array}{l}\text { Gradient } \\
\text { Correcte }\end{array}$ & & $\begin{array}{l}\text { Gradient- } \\
\text { Corrected + } \\
\text { Solvation }\end{array}$ \\
\hline $\begin{array}{l}{[\mathrm{Ni}(\mathrm{EDTA})]^{2-}} \\
{\left[\mathrm{NiN}_{2} \mathrm{O}_{4}\right]^{2-26}}\end{array}$ & $\begin{array}{l}\mathrm{N}_{\mathrm{AV}}=2.077 \\
\mathrm{O}_{\mathrm{AV}}=2.067\end{array}$ & $\begin{array}{l}\mathrm{N}_{\mathrm{AV}}=2.074 \\
\mathrm{O}_{\mathrm{AV}}=2.046\end{array}$ & & & & \\
\hline $\begin{array}{l}\text { "amenip" } \\
{\left[\mathrm{NiN}_{3} \mathrm{O}_{3}\right]} \\
{\left[\mathrm{Ni}(\text { onno })_{3}\right]^{-}}\end{array}$ & $\begin{array}{l}\mathrm{N}_{\mathrm{AV}}=2.081 \\
\mathrm{O}_{\mathrm{AV}}=2.076\end{array}$ & $\begin{array}{l}\mathrm{N}_{\mathrm{AV}}=2.119 \\
\mathrm{O}_{\mathrm{AV}}=2.031\end{array}$ & $\begin{array}{l}\mathrm{N}_{\mathrm{AV}}=2.068 \\
\mathrm{O}_{\mathrm{AV}}=2.063\end{array}$ & $\begin{array}{l}\mathrm{N}_{\mathrm{AV}} \\
2.210 \\
\mathrm{O}_{\mathrm{AV}} \\
2.099\end{array}$ & $=$ & $\begin{array}{l}\mathrm{N}_{\mathrm{AV}}=2.136 \\
\mathrm{O}_{\mathrm{AV}}=2.146\end{array}$ \\
\hline $\begin{array}{l}\text { "duwvam" } \\
{\left[\mathrm{NiN}_{4} \mathrm{O}_{2}\right]^{2+}}\end{array}$ & $\begin{array}{l}\mathrm{N}_{\mathrm{AV}}=2.086 \\
\mathrm{O}_{\mathrm{AV}}=2.170\end{array}$ & & & & & \\
\hline$\left[\mathrm{Ni}(\text { dien })_{2}\right]^{2+}$ & $\begin{array}{l}\mathrm{N}_{\mathrm{AV}}=2.164 \\
2.135,2.087\end{array}$ & $\begin{array}{l}\mathrm{N}_{\mathrm{AV}}=2.140 \\
2.110,2.059\end{array}$ & & $\begin{array}{l}\mathrm{N}_{\mathrm{AV}} \\
2.239 \\
2.205 \\
2.127\end{array}$ & $=$ & $\begin{array}{l}\mathrm{N}_{\mathrm{AV}}=2.202, \\
2.171,2.116\end{array}$ \\
\hline
\end{tabular}


Table S3. Structural Data available for the two series $\left[\mathrm{Pt}^{\mathrm{II}} \mathrm{Cl}_{n}\left(\mathrm{NH}_{3}\right)_{4-\mathrm{n}}\right]$ and $\left[\mathrm{Pt}^{\mathrm{II}} \mathrm{Cl}_{\mathrm{n}}(\mathrm{CO})_{4-\mathrm{n}}\right]$

\begin{tabular}{|c|c|c|c|c|}
\hline \multirow[t]{2}{*}{ Complex } & \multicolumn{2}{|l|}{$\mathrm{L}=\mathrm{NH}_{3}$} & \multicolumn{2}{|l|}{$\mathbf{L}=\mathbf{C O}$} \\
\hline & Pt-N (Å) & Pt-Cl (Å) & Pt-C $(\AA)$ & Pt-Cl (Å) \\
\hline$\left[\mathrm{PtCl}_{4}\right]^{2-}$ & & 2.299 & & 2.299 \\
\hline$\left[\mathrm{PtCl}_{3} \mathrm{~L}\right]^{-}$ & 2.088 & 2.291 & 1.825 & \\
\hline cis- $\left[\mathrm{PtCl}_{2} \mathrm{~L}_{2}\right]$ & 2.033 & 2.300 & 1.897 & 2.292 \\
\hline \multicolumn{5}{|c|}{ trans $-\left[\mathrm{PtCl}_{2} \mathrm{~L}_{2}\right]$} \\
\hline \multicolumn{5}{|c|}{$\left[\mathrm{PtClL}_{3}\right]^{+}$} \\
\hline$\left[\mathrm{PtL}_{4}\right]^{2+}$ & 2.048 & & 1.982 & 2.291 \\
\hline
\end{tabular}

Table S4. The Series $\left[\mathrm{Pt}^{\mathrm{II}}\left(\mathrm{NH}_{3}\right)_{\mathrm{n}} \mathrm{Cl}_{4-\mathrm{n}}\right]$

\begin{tabular}{|c|c|c|c|c|c|c|c|c|}
\hline \multirow[t]{2}{*}{ Complex } & \multicolumn{2}{|l|}{ Ida } & \multicolumn{2}{|c|}{ lda+solvation } & \multicolumn{2}{|l|}{ Gga } & \multicolumn{2}{|c|}{ gga+solvation } \\
\hline & $\mathrm{Pt}-\mathrm{Cl}$ & Pt-N & $\mathrm{Pt}-\mathrm{Cl}$ & Pt-N & $\mathrm{Pt}-\mathrm{Cl}$ & Pt-N & $\mathrm{Pt}-\mathrm{Cl}$ & Pt-N \\
\hline$\left[\mathrm{PtCl}_{4}\right]^{2-}$ & 2.321 & & 2.299 & & 2.383 & & 2.357 & \\
\hline$\left[\mathrm{PtCl}_{3} \mathrm{NH}_{3}\right]^{-}$ & 2.295 & 2.023 & & & 2.345 & 2.075 & 2.359 & 2.058 \\
\hline cis- $\left[\mathrm{PtCl}_{2}\left(\mathrm{NH}_{3}\right)_{2}\right]$ & 2.270 & 2.047 & 2.30 & 2.016 & 2.314 & 2.100 & 2.361 & 2.060 \\
\hline $\begin{array}{l}\text { trans- } \\
{\left[\mathrm{PtCl}_{2}\left(\mathrm{NH}_{3}\right)_{2}\right]}\end{array}$ & 2.285 & 2.011 & 2.292 & 2.027 & 2.332 & 2.052 & 2.355 & 2.072 \\
\hline$\left[\operatorname{PtCl}\left(\mathrm{NH}_{3}\right)_{3}\right]^{+}$ & 2.129 & 2.092 & 2.293 & 2.023 & 2.286 & 2.092 & 2.352 & 2.067 \\
\hline$\left[\mathrm{Pt}\left(\mathrm{NH}_{3}\right)_{4}\right]^{2+}$ & & 2.042 & & 1.978 & & 2.048 & & 2.091 \\
\hline
\end{tabular}


Table S5. The Series $\left[\mathrm{Pt}^{\mathrm{II}}(\mathrm{CO})_{\mathrm{n}} \mathrm{Cl}_{4-\mathrm{n}}\right]$

\begin{tabular}{|c|c|c|c|c|c|c|c|c|}
\hline \multirow[t]{2}{*}{ Complex } & \multicolumn{2}{|l|}{ lda } & \multicolumn{2}{|c|}{ Ida+solvation } & \multicolumn{2}{|l|}{ gga } & \multicolumn{2}{|c|}{ gga+solvation } \\
\hline & $\mathrm{Pt}-\mathrm{Cl}$ & Pt-C & $\mathrm{Pt}-\mathrm{Cl}$ & Pt-C & Pt-Cl & Pt-C & $\mathrm{Pt}-\mathrm{Cl}$ & Pt-C \\
\hline$\left[\mathrm{PtCl}_{4}\right]^{2-}$ & 2.321 & & 2.299 & & 2.383 & & 2.357 & \\
\hline$\left[\mathrm{PtCl}_{3} \mathrm{CO}^{-}\right.$ & $\begin{array}{l}2.317 \\
2.291\end{array}$ & 1.818 & 2.310 & 1.810 & $\begin{array}{l}2.372 \\
2.341\end{array}$ & 1.840 & $\begin{array}{l}2.369 \\
2.347\end{array}$ & 1.855 \\
\hline cis- $\left[\mathrm{PtCl}_{2}(\mathrm{CO})_{2}\right]$ & 2.274 & 1.868 & 2.304 & 1.902 & 2.318 & 1.902 & 2.275 & 1.952 \\
\hline $\begin{array}{l}\text { trans- } \\
{\left[\mathrm{PtCl}_{2}(\mathrm{CO})_{2}\right]}\end{array}$ & 2.303 & 1.903 & 2.309 & 1.881 & 2.351 & 1.933 & 2.361 & 1.904 \\
\hline$\left[\mathrm{PtCl}(\mathrm{CO})_{3}\right]^{+}$ & 2.28 & $\begin{array}{l}1.908 \\
1.891\end{array}$ & 2.30 & 1.932 & 2.34 & $\begin{array}{l}1.940 \\
1.961\end{array}$ & 2.327 & $\begin{array}{l}1.961 \\
1.908\end{array}$ \\
\hline$\left[\mathrm{Pt}(\mathrm{CO})_{4}\right]^{2+}$ & & 1.970 & & 1.907 & & 2.003 & & 1.950 \\
\hline
\end{tabular}


TableS7. Crystallographic and calculated geometric values for the series of compound with trans-influences.

\begin{tabular}{|c|c|c|c|c|c|c|c|c|}
\hline \multirow[t]{2}{*}{ Complex } & \multicolumn{2}{|c|}{$\begin{array}{l}\text { Crystallographic } \\
\text { Bond Lengths } \\
(\AA)\end{array}$} & \multicolumn{2}{|c|}{ LDA (VWN) } & \multicolumn{2}{|c|}{$\begin{array}{l}\text { Gradient } \\
\text { Corrected } \\
(\mathrm{BP} 86)\end{array}$} & \multicolumn{2}{|c|}{$\begin{array}{l}\text { Gradient } \\
\text { Corrected } \\
\text { +Solvation }\end{array}$} \\
\hline & cis & trans & cis & trans & cis & trans & cis & trans \\
\hline$\left[\mathrm{PtCl}_{4}\right]^{2-}$ & $\begin{array}{l}2.299 \\
(0.004)\end{array}$ & $\begin{array}{l}2.299 \\
(0.004)\end{array}$ & 2.321 & 2.321 & 2.383 & 2.383 & 2.357 & 2.357 \\
\hline${ }_{78,79}^{\left[\mathrm{PtCl}_{3} \mathrm{NH}_{3}\right]^{-}}$ & $\begin{array}{l}2.305 \\
(0.000)\end{array}$ & $\begin{array}{l}2.295 \\
(0.000)\end{array}$ & 2.299 & 2.324 & 2.351 & 2.372 & 2.356 & 2.364 \\
\hline$\left[\mathrm{PtCl}_{3} \mathrm{DMSO}\right]^{-}$ & $\begin{array}{l}2.296 \\
(0.005)\end{array}$ & $\begin{array}{l}2.316 \\
(0.007)\end{array}$ & 2.316 & 2.295 & 2.370 & 2.340 & 2.362 & 2.377 \\
\hline $\begin{array}{l}{\left[\mathrm{PtCl}_{3} \mathrm{PEt}_{3}\right]^{-}} \\
-\end{array}$ & $\begin{array}{l}2.300 \\
(0000)^{3}\end{array}$ & $\begin{array}{l}2.380 \\
(0000)\end{array}$ & 2.317 & 2.335 & 2.371 & 2.389 & 2.358 & 2.464 \\
\hline $\begin{array}{l}{\left[\mathrm{PtCl}_{3}\left(\mathrm{C}_{2} \mathrm{H}_{4}\right)\right]^{-}} \\
\text {(Zeise’s } \quad \text { Salt) } \\
80,81\end{array}$ & $\begin{array}{l}2.301 \\
(0.009)\end{array}$ & $\begin{array}{l}2.331 \\
(0.008)\end{array}$ & 2.309 & 2.294 & 2.365 & 2.345 & 2.357 & 2.384 \\
\hline$\left[\mathrm{PtCl}_{3} \mathrm{CO}\right]^{-82}$ & $\begin{array}{l}2.292 \\
(0.000)\end{array}$ & $\begin{array}{l}2.289 \\
(0.000)\end{array}$ & 2.317 & 2.291 & 2.372 & 2.340 & 2.364 & 2.350 \\
\hline
\end{tabular}

\footnotetext{
${ }^{1}$ Derived by the statistical analysis of 36 separate crystallographic observations of $\left[\mathrm{PtCl}_{4}\right]^{2-}$. The error quoted represents a confidence interval of $95 \%$ certainty on the mean.

${ }^{2}$ Derived by the statistical analysis of 7 separate crystallographic observations of $\left[\mathrm{PtCl}_{3}(\mathrm{DMSO})\right]^{2-}$. The error quoted represents a confidence interval of $95 \%$ certainty on the mean.

${ }^{3}$ This error represents a crystallographic calculation of one standard deviation and not a statistically derived error.
} 
Table S8. Co-X bond lengths in some complexes exhibiting structural trans-influences

\begin{tabular}{c|lllll}
\hline $\begin{array}{c}\text { Complex and } \\
\text { Reference }\end{array}$ & $\begin{array}{c}\text { Experi- } \\
\text { mental }\end{array}$ & \multicolumn{1}{c}{ LDA } & $\begin{array}{c}\text { Gradient } \\
\text { Corrected }\end{array}$ & $\begin{array}{c}\text { LDA+ } \\
\text { Solvaton }\end{array}$ & $\begin{array}{c}\text { Gradient } \\
\text { Corrected } \\
\text { +Solvation }\end{array}$ \\
\hline$\left[\mathrm{Co}\left(\mathrm{NH}_{3}\right)_{5} \mathrm{CH}_{3}\right]^{2+66}$ & $\mathrm{~N}_{\text {trans }}=2.105$ & $\mathrm{~N}_{\text {trans }}=2.070$ & $\mathrm{~N}_{\text {trans }}=2.016$ & $\mathrm{~N}_{\text {trans }}=2.017$ & $\mathrm{~N}_{\text {trans }}=2.101$ \\
& $\mathrm{~N}_{\text {cis }}=1.973$ & $\mathrm{~N}_{\text {cis }}=1.941$ & $\mathrm{~N}_{\text {cis }}=2.165$ & $\mathrm{~N}_{\text {cis }}=1.919$ & $\mathrm{~N}_{\text {cis }}=1.985$ \\
& $\mathrm{CH}_{3}=1.979$ & $\mathrm{CH}_{3}=1.943$ & $\mathrm{CH}_{3}=2.000$ & $\mathrm{CH}_{3}=1.935$ & $\mathrm{CH}_{3}=1.987$ \\
{$\left[\mathrm{Co}\left(\mathrm{NH}_{3}\right)_{5} \mathrm{NHCOCH}_{3}\right]^{2+67}$} & $\mathrm{~N}_{\text {trans }}=1.994$ & $\mathrm{~N}_{\text {trans }}=1.971$ & $\mathrm{~N}_{\text {trans }}=2.075$ & $\mathrm{~N}_{\text {trans }}=1.940$ & $\mathrm{~N}_{\text {trans }}=2.006$ \\
& $\mathrm{~N}_{\text {cis }}=1.963$ & $\mathrm{~N}_{\text {cis }}=1.946$ & $\mathrm{~N}_{\text {cis }}=2.003$ & $\mathrm{~N}_{\text {cis }}=1.919$ & $\mathrm{~N}_{\text {cis }}=1.978$ \\
& $\mathrm{~N}_{\text {amide }}=1.911$ & $\mathrm{~N}_{\text {amide }}=1.900$ & $\mathrm{~N}_{\text {amide }}=1.943$ & $\mathrm{~N}_{\text {amide }}=1.884$ & $\mathrm{~N}_{\text {amide }}=1.938$ \\
& & $\mathrm{~N}_{\text {trans }}=2.065$ & $\mathrm{~N}_{\text {trans }}=2.165$ & $\mathrm{~N}_{\text {trans }}=2.021$ & $\mathrm{~N}_{\text {trans }}=2.100$ \\
& & $\mathrm{~N}_{\text {cis }}=1.941$ & $\mathrm{~N}_{\text {cis }}=2.006$ & $\mathrm{~N}_{\text {cis }}=1.918$ & $\mathrm{~N}_{\text {cis }}=1.982$ \\
& & $\mathrm{H}=1.443$ & $\mathrm{H}=1.451$ & $\mathrm{H}=1.452$ & $\mathrm{H}=1.461$ \\
\hline
\end{tabular}




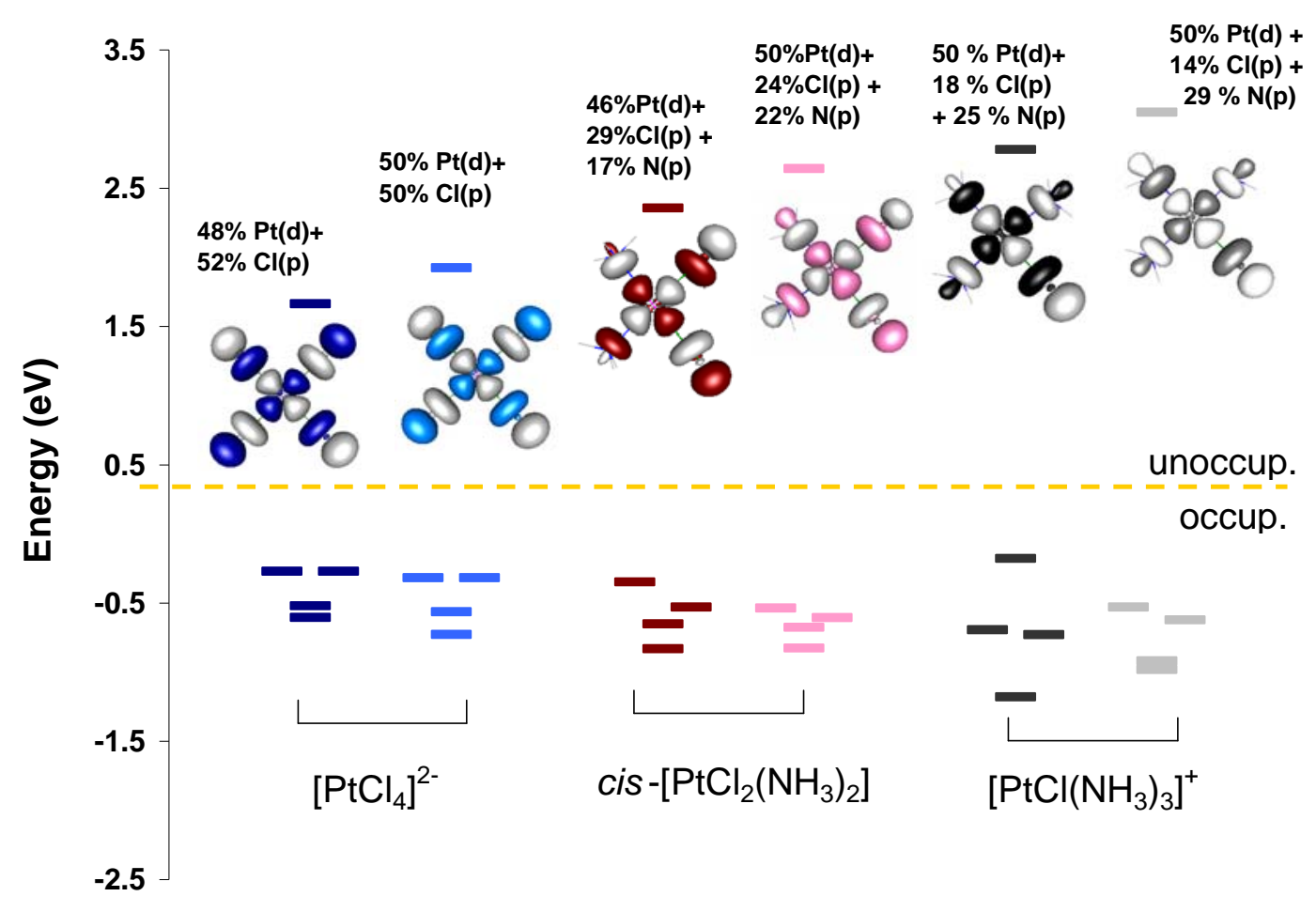

Figure S5. d-based molecular orbitals for the series of compounds $\left[\mathrm{PtCl}_{4}\right]^{2-}$, cis$\left[\mathrm{PtCl}_{2}\left(\mathrm{NH}_{3}\right)_{2}\right]$ and $\left[\mathrm{PtCl}\left(\mathrm{NH}_{3}\right)_{3}\right]^{+}$. The LUMO (Fe dx2-y2, orbital is plotted). Gas phase calculated energy levels are to the left, solvated orbitals are to the right. The energies of each set of orbitals has been set so that the average of the five orbitals is zero. 


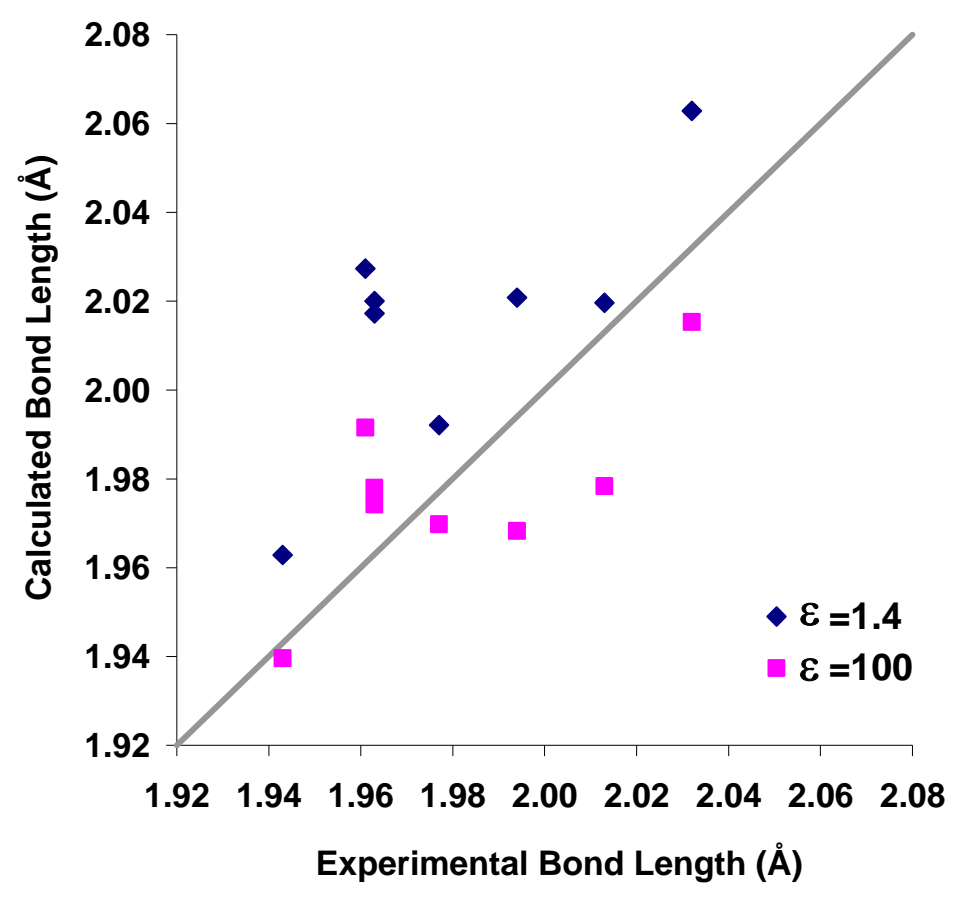

Figure S6. A comparison of the experimental versus calculated bond lengths for the cobalt hexa-amines. 
(1) Hocking R. K.; Hambley T. W. Inorg. Chem. 2002, 21, 2660-2666.

(2) Brunner H.; Ludi A.; Raselli A.; Bürgi H. -B. Helv. Chim. Acta. 1996, 79, 1607-1610.

(3) Yoneda H. unpublished data.

(4) Henrick K.; McPartlin M.; Munjoma S.; Owston P. G.; Peters R.;

Sangokoya S. A.; Tasker P.A. J. Chem. Soc., Dalton Trans. 1982, 225-227.

(5) Bernhardt P. V.; Lawrence G. A.; Hambley T. W. J. Chem. Soc., Dalton

Trans. 1989, 1059-1065.

(6) Bernhardt P. V.; Jones L. A. J. Chem. Soc., Dalton Trans 1998, 1757-

1761.

(7) Hambley T. W.; Searle G. H.; Snow M. R. Aust. J. Chem. 1982, 35, 1285-

1295.

(8) Ishi M.; Umehara M.; Harada K.; Nakahara M. Bull. Chem. Soc. Jpn. 1987, 60, 2683-2685.

(9) Munk V. P.; S., C.; Fenton R. R.; Hocking R. K.; Hambley T.W. Aust. J. Chem. 2002, 55, 523-529.

(10) Hambley T. W. J. Chem. Soc., Dalton Trans. 1986, 565.

(11) Barefield E.F.; Freeman G. M.; Derveer D. G. Inorg. Chem. 1986, 25,

552.

(12) Hancock R. D.; Dobson S. M.; Evers A.; Wade P. W.; Ngwenya M. P.; Boeyens J. C. A.; Wainwright K. P. J. Am. Chem. Soc. 1988, 110, 2788.

(13) Porai-Koshits M. A.; Kukina G. A. Izv. Akad. Nauk. SSSR, Ser. Khim.

1993, 1492.

(14) Billo E. J.; Connolly P. J.; Sardella D. J.; Jasinski J. P.; Butcher R. J. Inorg. Chim. Acta. 1995, 230, 19.

(15) Barefield E. F.; Chueng D.; Vanderveer D. G.; Wagner F. Chem.

Commun. 1981, 302.

(16) Prasad L.; McAuley A. Acta Cystallogr., Sect. C (Cr. Str. Comm.) 1983, 39, 1175.

(17) Barefield E. K.; Bianchi A.; Billo E. J.; Connolly P. J.; Paoletti P.;

Summers J. S.; Van Derveer D. G. Inorg. Chem. 1986, 25, 4197.

(18) McAuley A.; Subramanian S.; Whitcombe T. W. Chem. Commun. 1987,

539.

(19) Prasad L.; Nyburg S. C.; McAuley A. Acta Crystallogr., Sect C. (Cr. Str. Comm.) 1987, 43, 1038.

(20) Adam K. R.; Antolovich M.; Brigden L. G.; Leong A. J.; Lindoy L. F.; Baillie P. J.; Uppal D. K.; McPartlin M.; Shah B.; Proserpio D.; Fabbrizzi L.; Tasker P. A. J. Chem. Soc., Dalton Trans. 1991, 2493.

(21) Chen L.; Cotton F. A. J. Mol. Struct. 1998, 470, 161-166.

(22) Allinger N. L. J. Am. Chem. Soc. 1977, 99, 8127-8135.

(23) Bray M. R.; Deeth R. J.; Paget V. J.; Sheen P. D. Int. J. Quantum Chem. 1996, 61, 85-91.

(24) McDougall G. J.; Hancock R. D. J. Chem. Soc., Dalton Trans. 1980, 654. 
(25) Scharzenbach G.; Bürgi H-B.; Jenson W. P.; Lawrence G.A.; Monsted L.; M., S. A. Inorg. Chem. 1983, 22, 4029.

(26) Nesterova Ya. M. Koord. Khim. 1984, 10, 129. 